\title{
DEVELOPMENT OF CAMPUS SPACE NAVIGATION AND GUIDE SYSTEM
}

\author{
Yan-Chyuan Shiau \\ Chung Hua University, Hsin-Chu, Taiwan \\ ycshiau@ms22.hinet.net \\ Tsung-Pin Tsai \\ Chung Hua University, Hsin-Chu, Taiwan \\ winnie.bear@msa.hinet.net \\ Jia-Chu Lin \\ Chung Hua University, Hsin-Chu, Taiwan \\ sfh40413@yahoo.com.tw \\ Chih-Tsun Hsiao \\ Chung Hua University, Hsin-Chu, Taiwan \\ chun.chun888@msa.hinet.net
}

\begin{abstract}
For larger enterprises usually occupying vast areas, visitors usually need to take long time to reach the objective location in an unfamiliar environment. SQL Server, ASP.net, PDA, GPS, Papago Sdk, wireless communication and windows environment are used in this study to develop "Space Navigation and Guide System”. The system integrates personnel and space information for the campus. When related data are input, the system will list all matched faculty information. It will navigate to the entry of the building where the chosen faculty located by GPS. The system will guide to exact floor and room by WiFi system inside building. This can significantly save the exploring time of the visitor, improve the space service and promote the enterprise image. User can use browser to preview 3D animation for the interesting spots. This can help visitors to become familiar the new environment before their visit.
\end{abstract}

\section{KEYWORDS: GPS, WiFi, database, PDA}

\section{INTRODUCTION}

\section{Motives and Objectives}

The application of space location in Taiwan and other countries usually relies on the GPS outside the building, because the satellite signal is blocked by the building and, thus, navigation is not available indoors. Lager companies, institutions or schools cannot provide visitors with more complete space information and convenient navigation service due to the lack of an effective guide system (Fang H. A., 2005). Nevertheless, the combination of GPS, wireless network and WiFi technology will provide a rapid campus visual navigation and guide service that is friendlier to the user. 
When buying a house, many customers must come to the site personally to compare and discuss before they can make the decision. The real estate broker in early days could only provide 2D drawings and photos and it was difficult for the buyer to fetch adequate information there from. So customers must personally go back and forth between houses and become travel-worn. Currently, some real estate brokerage companies have used 3D Space software and photo-synchronized 3D animation to provide a 720-degree image that enables their customers to view the status and environment of the targeted house via the internet. In this way, the buyer does not need to go out to fetch the spatial layout of the house and can conserve their time effectively. This is really good news to the buyer of the house (3D Space, 2008). The Google Map (Wikipedia, 2001) is an electronic map provided by Google. It covers detailed local satellite photos and can provide vector maps, satellite photos and terrain views. Google Earth further provides 3D models that show the street views, more satellite photos and GPS location function. It allows the user to fetch the real scene and provide a very convenient environment for the enquiry of map information. Unfamiliarity and fear of the new environment will be reduced if the user can become familiar with it first using 3D images via the Internet. This study is trying to build space navigation and guide system and provide a rapid, convenient and precise navigation and guide tool by integrating the GPS system, wireless communication system and 3D animation.

\section{LITERATURE OVERVIEW}

\section{GPS system}

The development of GPS is lead by the Defence Department of the United States. It is partially transferred to the private sector for the applications of navigation positioning, precise measurement and standard time. The navigation positioning technology has a wide applications and every one can use it to find correct position, speed and time of a carrier. The worst disadvantage of the GPS is that it must be within the accessibility of the satellite. So positioning cannot be carried out in a room or building because the satellite signal cannot be transmitted directly to the equipment of the client. GPS is an outdoor positioning technology.

\section{PAPAGO SDK}

PaPaGo SDK provides GIS geographical information system with a high-performance map core and can be deployed in conjunction with the space information of the map manufacturer. The developer of the system can focus on the analysis of the data, reveal of the result and support of the decision. One can significantly shorten the time-to-market, manpower and cost. PaPaGo SDK uses the standard COM interface and can support various development environments, including $\mathrm{C}, \mathrm{C}++$, Visual Basic, C\#, and so on. This software allows rapid integration of components to build a system that meets the requirements. The development of the electronic map core involves some information engineering technique, including design of a large database, construction of optimized search engine, calculation of the best route and coordinate transformation (Jian L. Y. and Cai C. Z., 2001).

PaPaGo SDK contains three modules: Display, Find and Routing. The Display module provides the functions of open/closure of the map, zooming of the map, translation, full screen and rotation. The Find module provides the functions of acronym search, GPS inquiry 
and intersection search. The Routing module provides the functions of the best route search, end-to-end distance and multipoint routing (Jian L. Y. et al., 2002).

\section{D system}

The 3D system is developed by 3D Space. The image taken by using a 3D camera is converted to a 3D real scene using the conversion software. It can be displayed panoramically at 360 degrees x 360 degrees. The panoramic video of 3D Space provides a 360-degree view and there is no dead space no matter whether viewing from above, below, right or left. The display can be upgraded to 3D real scenes using only a little bit of resources. This is attractive to many customers and, thus, increases the closure rate and sales performance easily (Wikipedia, 2001). The size of the file is small, about $30 \mathrm{~KB}$ to $300 \mathrm{~KB}$. The user can manually adjust the resolution of the display. 3D Space provides Java Applet, so the user can view the application without the need to install additional software. It actually produces the effect of the virtual reality and space guide.

\section{Wi-Fi and LBS platform}

LBS (Location Based Services) is an "addressing service” or "mobile location-based service”. It is an application service that combines the latest information and communication technology, including the technologies of the mobile communication system or handheld device, geographical information system and the Internet. LBS implements the positioning and tracking function using a mobile communication system or handheld device. It combines the information of the geographic information system to provide application service via the Internet (Lin R. M., 2008).

\section{PLANNING OF CAMPUS NAVIGATION AND GUIDE SYSTEM}

\section{Analysis of the system function}

The information management system must take the completeness and running efficiency of the system into account. To do the system function analysis, the function to be used, the datasheet to be processed and the field, property and mutual relationship must be analyzed to reduce the cost of modification and maintenance in the future. This study analyzes four operating modules: Keyword search, Structure Search, Activity Search, and Navigation \& Guide.

\section{Keyword search operation module}

The keyword search operation contains following sub-function:

1. Maintenance of the search target: It is provided to search the name, space number, office number, extension phone number, mobile phone number and fax number.

2. Maintenance of search conditions: It provides precise and fuzzy searches. The user can input a complete data to make exact march search or input a fraction of the data to make partial match search. 
3. Maintenance of the search string: The user inputs the string to be searched to make comparison and query.

\section{Structure search operation module}

The structure search operation contains following sub-function:

1. Maintenance of the teaching unit: This structure divides the teaching unit into two levels: college and departments/institutes subordinate to the college. When a college is selected, the departments/institutes of the selected college will be listed. When a department/institute is selected, all teachers and staff of the selected department/institute will be listed for query.

2. Maintenance of the administrative unit: This structure divides the teaching unit into two levels: Level 1 (incl. each office and center) and Level 2 (each section). When a Level-1 unit is selected, all offices and centers subordinate to the selected unit will be listed. When a Level-2 unit is selected, the data of the faculty subordinate to the unit will be listed for query.

\section{Activity search operation module}

Maintenance of the activity name provides information, space and responsible person related to the organization of an activity or workshop in the college.

\section{Navigation and guide operation module}

The navigation and guide operation contains following sub-function:

1. Maintenance of outdoor GPS navigation: When the user has selected the person to be visited, visitor can read the room number of the selected person and retrieve the building where the person is located. Then the system acquires the longitude and latitude of the point at which the user currently stays and the longitude and latitude of the entrance of the building. The best route between the two points will be calculated for outdoor navigation and to help the visitor to navigate to the entrance of the building.

2. Maintenance of indoor LBS guide: When the visitor uses the GPS navigation function and navigates to the entrance of the building, the indoor LBS system will guide the user to the floor and room where he/she wants to go.

\section{Structure of the system database}

This study uses the ER/Studio tool to establish E-R Model. The database is planned by following the normal steps to make it more efficient and easily maintainable. It is also helpful to avoid repetition or incompatibility in the design and implementation phases of the database.

The data module of this study contains 9 datasheets for building data, floor data, space data, activity data, animation scheme data, activity space data, college data, department/institute data and faculty data. 


\section{Creation of the initial data}

This study uses the SQL syntax to feed related data in the database line by line to create more than 3,000 initial data for developing, debugging and training of the system (Table 1).

Table 1: The content and quantity of the data that have been pre-input in the system

\begin{tabular}{lc}
\hline Data Type & Quantity \\
\hline Number of buildings & 15 \\
\hline Number of floors & 110 \\
\hline Number of rooms & 1,085 \\
\hline College and Level-1 office/center data & 29 \\
\hline Department/institute and Level-2 unit data & 119 \\
\hline Faculty data & 487 \\
\hline Activity project data & 9 \\
\hline GPS location data & 149 \\
\hline Detailed space data & 1,059 \\
\hline Total & 3,062 \\
\hline
\end{tabular}

\section{CAMPUS SATELLITE LOCATION, LBS CONSTRUCTION AND 3D ANIMATION SETUP}

\section{Campus satellite location}

This study uses a mobile phone with the GPS location function to collect fixed-point data at different positions on the campus. Then the measured data are used to draw the plan of the campus and mark the position of the road. These data are also used to guide the user to the nearest distance and precise position and set the decision point for the entrance of the building, so that the system can plan the guidance route rapidly. This study divided the campus into 17 areas for the measurement (Figure 1) and each of the four corners of a building is used as the measuring point. Then an extensive location procedure is carried out.

\section{LBS construction and testing}

This study uses SMC CWBR14-G2 and ZyXEL NBG460N as the indoor WiFi location AP. A total of eight wireless base stations are constructed on the second and third floors of the College of Architecture for synchronous receiving and identification. The LBS location 
system needs at lest 4 Applications for horizontal and vertical location. The floor plans are shown in Figure 2.

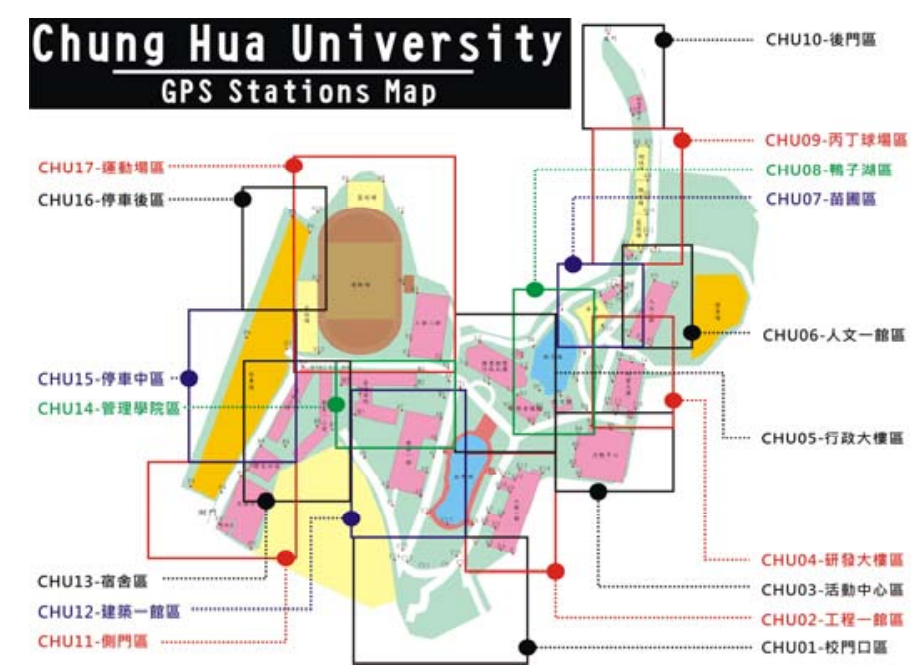

Figure 1: GPS measuring areas in the campus of Chung Hwa University

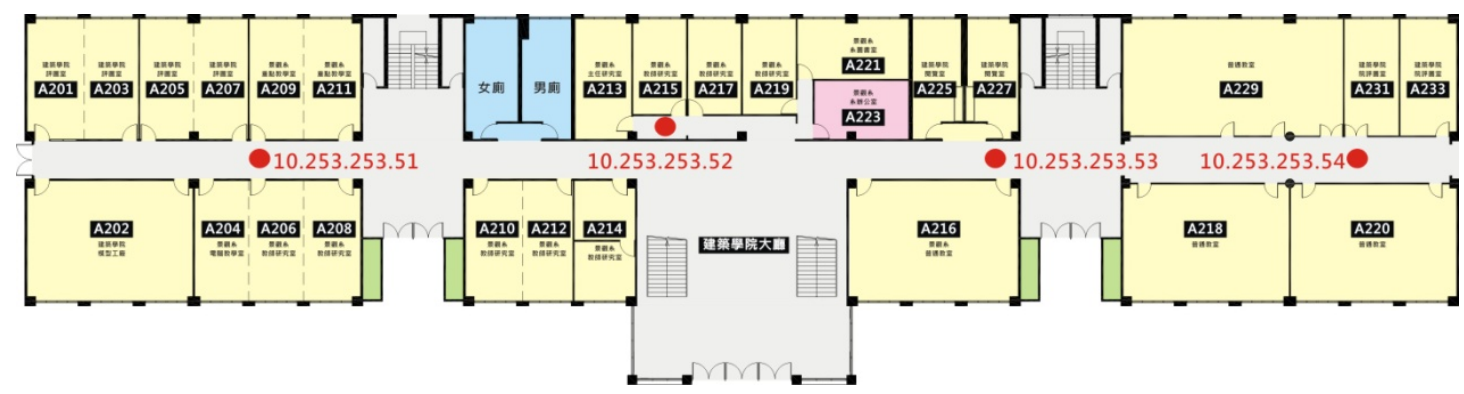

Figure 2: AP scatter on the second floor of the College of Architecture

\section{D animation setup}

This study uses the 3D animation setup system developed by 3D Space. After two photos are taken at 180 degrees using a 3D camera, the image files are converted to 3D real scenes using the conversion software (Figure 3) and the image can be displayed panoramically at 360 degrees x 360 degrees.

\section{OVERVIEW OF THE SYSTEM OPERATION}

\section{Keyword search}

The keyword search operation contains the "Search Target", "Search Conditions" and "Search String". The search target of personal data contains the name, mobile number, room number and telephone number of the office (as shown in Figure 4). When the target string is keyed-in and the "Start" button is pushed, the matched data will be listed below the display for user to pick the interesting person. The system will then activate the GPS function to guide visitor to the entrance of the building where the target person located. 


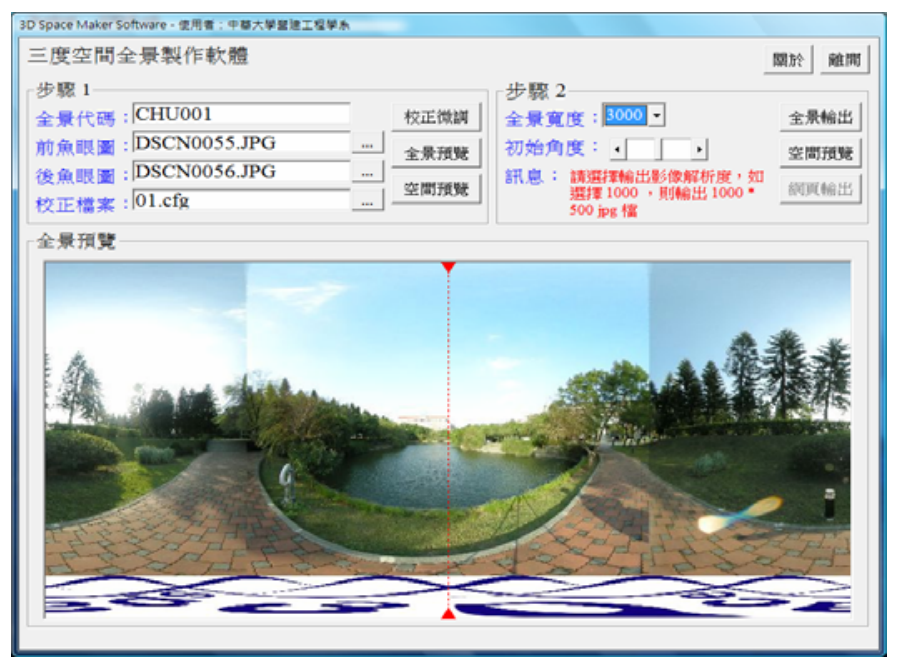

Figure 3: Software for production of 3D panoramic view

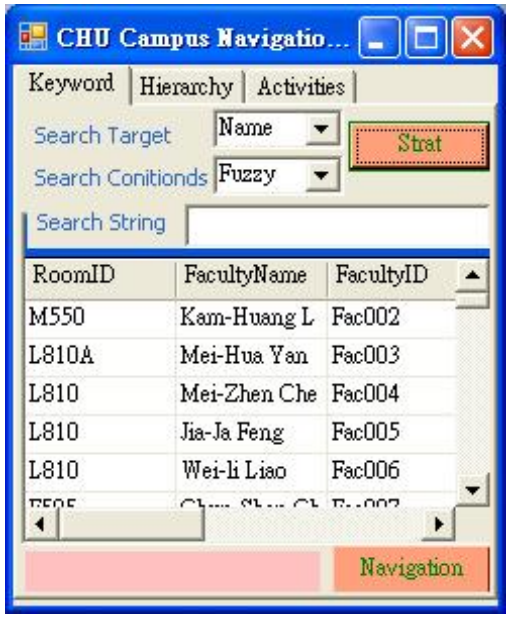

(a) Search by name

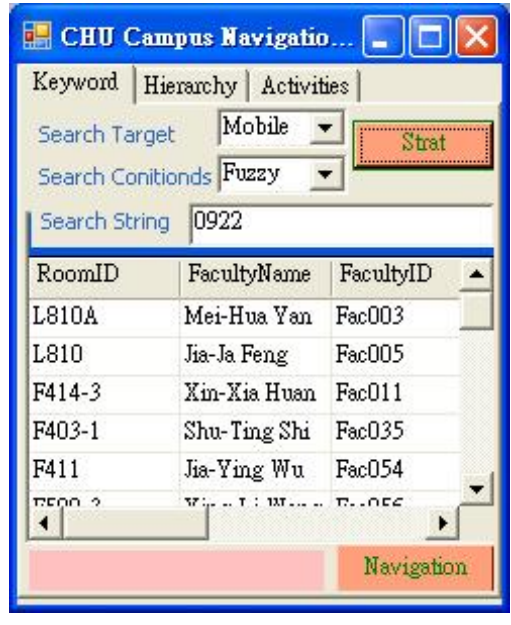

(b) Search by mobile number

Figure 4: Keyword search display

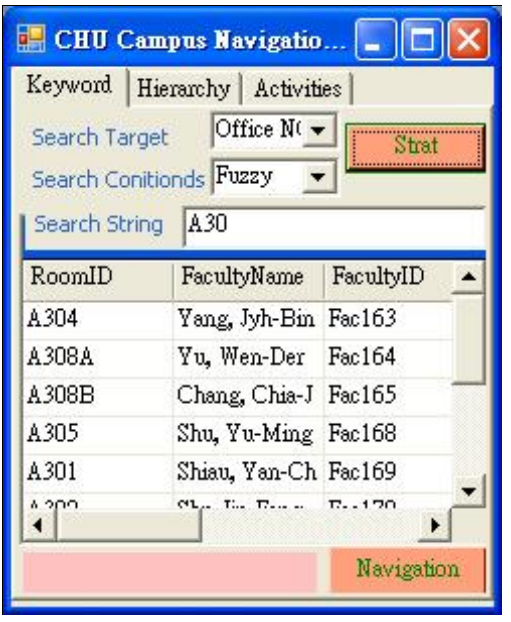

(c) Search by room id

\section{Hierarchy search}

The hierarchy search operation of the system contains the "Teaching Unit Search" and "Administrative Unit Search". The system will display the name of each college of the teaching unit when the "Teaching Unit" is selected. When a college is selected, the names of all departments and institutes of the selected college will appear on the right of the screen. When a department/institute is selected, the data of all teachers and staffs of the selected department/institute will appear at the bottom as shown in Figure 5. When the user picks "Administrative Unit" on the screen, the system will display the name of each Level-1 administrative unit. When an office/center is selected, the names of all Level-2 unit of the selected office/center will appear on the right of the screen. When a Level-2 unit is selected, the data of all staffs of the selected unit will appear at the bottom for user to pick the interesting person (as shown in Figure 6). 


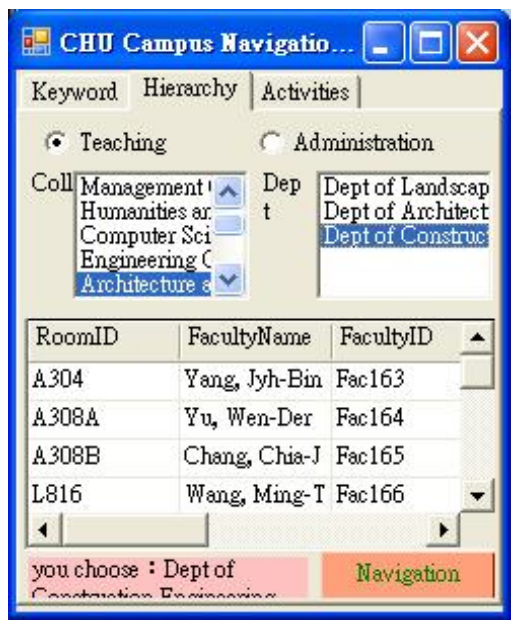

Figure 5: Hierarchy search - teaching unit

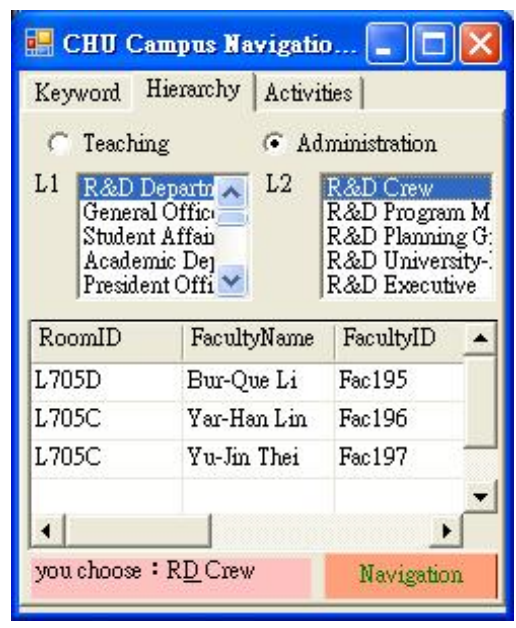

Figure 6: Hierarchy search - administration unit

\section{Activity search}

The activity search operation of the system contains the "Activity Name", "Leader" and "Venue". When an activity item is selected, the system will automatically display the responsible person and organizer of the selected activity. (Figure 7)

\section{Navigation}

When the visitor finds the data of the person to be visited and press the "Navigation" button, the system will execute the outdoor navigation. Figure 8 shows the navigation path from the gate of the university to the entrance of the Architecture Building. When the visitor arrives at the entrance of the building, the system will switch to the LBS indoor location function to take the visitor to the floor and office that he/she wants to go. (Figure 9)

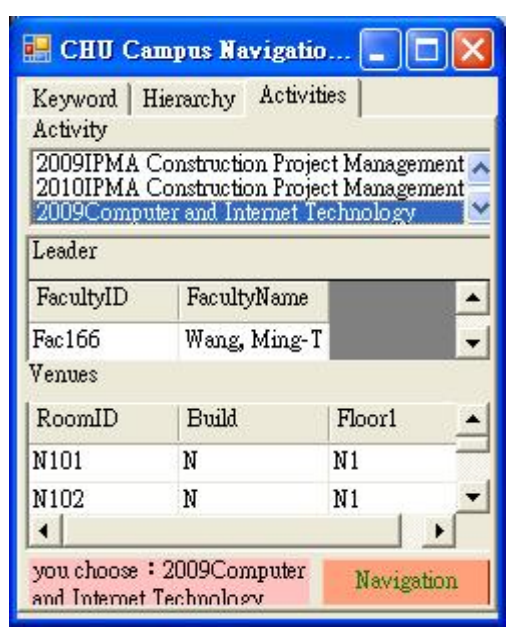

Figure 7: Activity search display
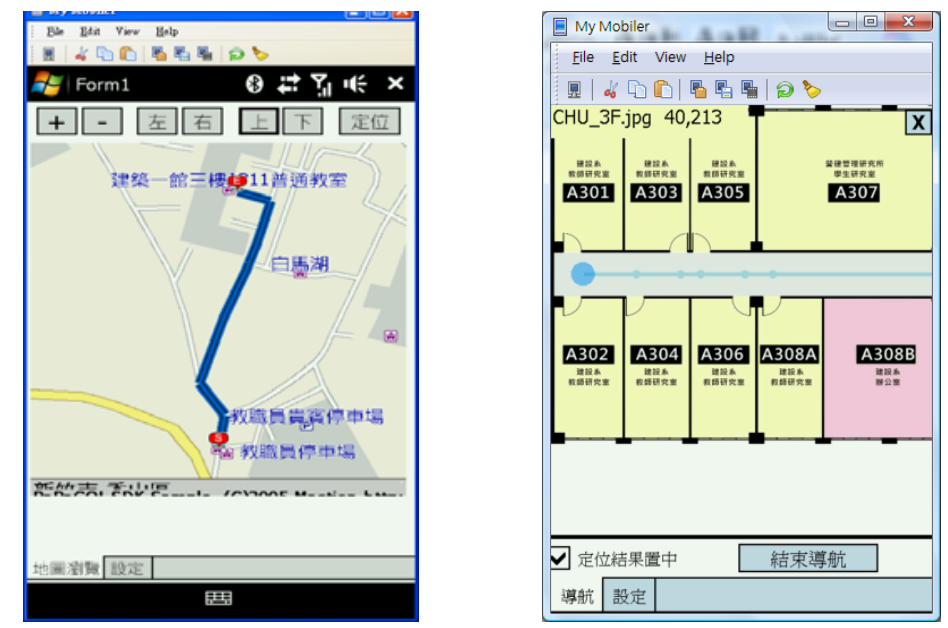

Figure 8: Outdoor navigation display Figure 9: Indoor guide display 


\section{Guidance}

This study surveys, maps and sets up the floor plan of the campus for webpage query (as shown in Figure 10). Selecting a scenic spot in the campus will display the day or night scene of the selected spot. (Figure 11 and Figure 12)

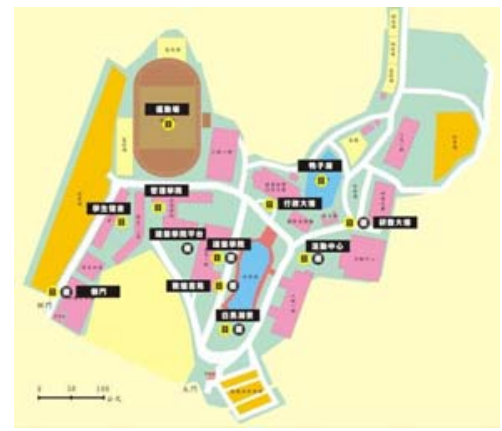

Figure 10: 3D view spots of the campus navigation system

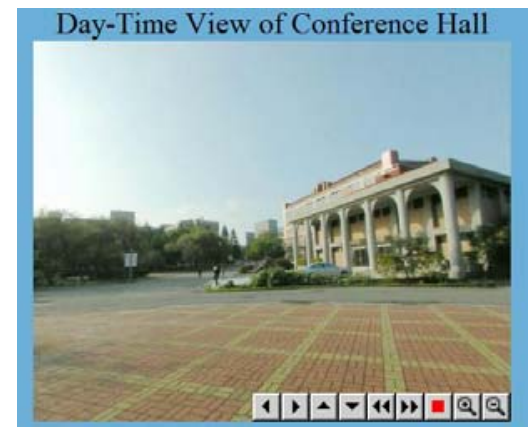

Figure 11: Day-time 3D view of the system

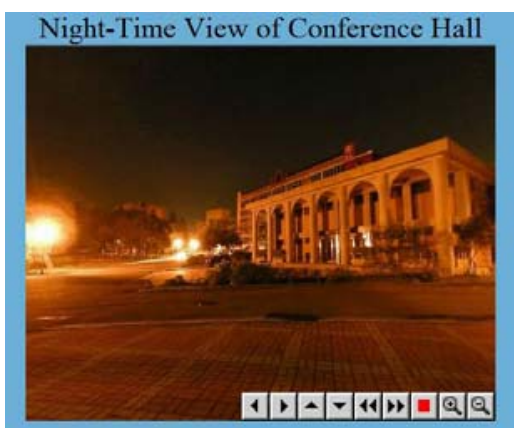

Figure 12: Night-time 3D view of the system

\section{CONCLUSION}

Though the navigation system is commonly used around the world, it usually provides the GPS outdoor location function rather than indoor location. Thanks to the population of Wi-Fi applications, using LBS for indoor location can significantly increase the efficiency of the location and help the user to find the targeted space easily. 3D guidance of the street scene has been available in Google map. The real estate brokers have started displaying the targeted house in $3 \mathrm{D}$ animation. It is very helpful to become familiar with the environment if scenic spots can be viewed in 3D animation on the Internet.

In addition to the campus space navigation and guide system, this study builds a database for the campus space and personnel affairs in order to improve the management capability. For people who have never visited the campus, 3D space guide page will be very helpful for them to become familiar with the environment. It can also provide the day and night scenes in different seasons. The following benefits will be acquired by doing so:

1. The campus navigation and guide system allows the person who visits the campus for the first time to find the room number of the person to be visited by searching his/her name, 
extension phone number or mobile phone number, or using the structure of the teaching and administrative systems.

2. The visitor can be guided to the entrance of the building with the help of the GPS navigation function. Indoors, the visitor will be taken to the room where he/she wants to go with the help of the Wi-Fi navigation function.

3. With the 360-degree animation, the user can use the browser to preview all scenic spots in the campus for their day and night scenes in different seasons. This allows the user to become familiar with the space in advance.

4. The activity project system module of this study can be used as a guide tool for academic workshops and activity projects.

5. This study can be used as a reference for the navigation and guidance in a large space, including large institutions, golf courses, stadiums or large international events. The system in this study allows the users who come from different countries in the world and are unfamiliar with the environment to search rapidly using this tool.

\section{REFERENCES}

Fang H. A. (2005) “The U-based Campus Guide system that combines GPS and RFID”, Master Essay, Dept. of Computer and Communication Engineering, Ming Chuan University.

Three Dimensional Space Network Technology Co., Ltd. (2008) “3D Space Software Description”, available at: http://www.3dspace.com.tw.

Wikipedia, (2001) “Google Map”, available at: http://zh.wikipedia.org.

Jian L. Y. and Cai C. Z., (2001) "Setup of the PaPaGo! SDK GIS System - Theory and Practice”, Maction Technologies, Inc.

Jian L. Y., Cai C. Z., and Wu J. W. (2002) "Development Practice of PaPaGo! SDK Mobile GIS System”, Maction Technologies, Inc.

Lin R. M. (2008) “Status Quo of LBS Technology Development”, Committee of Communications Industry Development, MOEA. 